\title{
Enhanced PAPR in OFDM without Deteriorating BER Performance
}

\author{
Spyridon K. Chronopoulos, Giorgos Tatsis, Vasilis Raptis, Panos Kostarakis \\ Physics Department, University of Ioannina, Ioannina, Greece \\ E-mail:schrono@cc.uoi.gr, \{gtatsis,vraptis\}@grads.uoi.gr,kostarakis@uoi.gr \\ Received November 14, 2010; revised February 9, 2011; accepted February 11, 2011
}

\begin{abstract}
Orthogonal frequency division multiplexing (OFDM) is vastly used in wireless networks. Its superiority relies on the fact that information can be split in large amount of frequencies. Each frequency is called information subcarrier. OFDM exhibits excellent annotation in channel fades and interferers as only a few subcarriers can be affected and consequently a small part of the original data stream can be lost. Orthogonality between frequencies ensures better spectrum management and obviates the danger of intersymbol interference. However, an essential problem exists. OFDM systems have high peak to average power ratio. This implies large fluctuations in signal power, ending up in increasing complexity of ADCs and DACs. Also, power amplifiers must work in a larger linear dynamic region. In this paper we present two new techniques for reducing Peak to Average Power Ratio (PAPR), that can be added in any OFDM system and we compare them with other existing schemes.
\end{abstract}

Keywords: OFDM, Convolutional Encoding, QPSK, Zero padding, IFFT, Cyclic Prefix, ISI, PAPR, Soft Reduction, $\mu$-Law Algorithm, Clipping Ratio (CR), Peak Ratio (PR), BER, CCDF, $\mu$ LSR, $\mu$ LaCP

\section{Introduction}

Orthogonal Frequency Division Multiplexing (OFDM) has been distinguished between other types of data transmission and reception schemes, for its excellent tolerance towards multipath fading and for supporting even higher data rates. OFDM has been a primary part of interest in many scientific researches and it has been included and implemented in various standards and application fields. Digital Audio Boadcasting (DAB), Terrestrial Digital Video Broadcasting (DVB-T), Wireless Local Area Network (WLAN - IEEE 802.11), High-Performance LAN type 2 (HIPERLAN/2), Broadband Wireless Access (BWA - IEEE 802.16), Mobile Broadband Wireless Access (802.20), WiMAX, Broadcast Radio Access Network (BRAN), Digital Subscriber Lines (DSL) and Multimedia Mobile Access Communication (MMAC) have all adopted OFDM [1].

The key feature of splitting data in various orthogonal information carriers along with the fact of introducing a guard band called cyclic prefix for avoiding ISI, characterizes a strong candidate transceiver for all future wireless applications. The main drawback is Peak to Average Power Ratio. This essential problem of subcarriers power fluctuation imposes undesirable complexity in Digital to Analog converters as they must operate in a wider dynamic range. Also the power amplifier, located on the transmitter part, must operate in a very large linear region for preventing spectral growth and consequently out-of-band noise. All previous demands relevant to existence of high PAPR, increase the overall cost of an OFDM system.

Many schemes for reducing PAPR have been proposed and are worth mentioning not only for their innovations but also due to hard work that appears to have been done by all authors. Clipping is very simple and has a quick implementation [2]. Unfortunately it causes outof-band radiation. Even if digital filtering is used for reducing radiation [3] which is very proper to do, BER deteriorates. Constellation shaping using SLM method in conjunction with Hadamard code [4] offers good results but complexity of this method is relatively high compared to others, like Low Complexity Technique which utilizes simple algorithm [5]. The latest still requires magnifier in receiver. Also in-depth BER performance is not mentioned. But, we must not omit the fact that its PAPR performance is fine. Another scheme is PAPR reduction with Huffman Coding [6] but it introduces the 
necessity of transmitting the encoding table to receiver. Even if bandwidth will not be affected, a serious drawback remains. System complexity is high. Another excellent idea is about recovering the clipped part of the OFDM signal [7], but it has restrictions, like trading-off between low CR and increasing the amount of the copied signal which in turn introduces redundancy in the transmitted data. Using a root companding transform technique [8] still requires expander in the receiver and exhibits good trade-off between PAPR and SER. SER Performance appears to be good but not innovative. Other technique using combined interleaving and companding [9] exhibits good CCDF performance but introduces the necessity of $\mathrm{k}$ interleavers in transmitter's part. Also side information must be sent to receiver containing identities of corresponding interleavers. This deteriorates simplicity of system design.

The first part of our work involved with the study of selected companders and was focused especially in two already known schemes which are soft reduction and $\mu$-algorithm. We selected these as they are simple techniques compared to others. We didn't use the expanded parts of these algorithms in the receiver in order to avoid overall complexity. Then we conducted various simulations ending up in finding two new strong candidates for PAPR reduction without deteriorating BER system performance. In the third part of our study final simulations of an OFDM system (up to 8192 IFFT subcarriers) were conducted. This system was consisted of a convolutional encoder [10] and a viterbi decoder along with other blocks.

This paper is split into six sections. In the second section details are given about the OFDM system that we used as a basic test platform for our proposed schemes with the presence of an AWGN channel. In the third section we analyze the behaviour of the new compressor that is composed by a soft reduction algorithm and $\mu$-compander in serial mode. Fourth section exhibits the behavior of a compression system that uses function of subtraction. This system removes the complex $\mu$-Law compander input signal, from its compressed output. Fifth section compares these new techniques with others in terms of PAPR. Sixth section concludes paper while giving future goals of our study.

\section{Simulation Platform Overview}

Our platform which is used as a basic simulation testbed, forms an OFDM system. All system delays were computed in order to apply a perfect synchronization between transmitter and receiver. Also, each time we added or removed blocks we calculated the power characteristics of the new generated OFDM signal, in order our simulations to produce the highest possible accurate results. Transmitter system under test was constituted of a random generator, a convolutional encoder, a QPSK modulator, a serial to parallel converter, an oversampling procedure using double zero padding, an IFFT block, a cyclic prefix generator and an unbuffering procedure. All inverse computations were implemented in receiver's part. Specifically for implementing convolutianal encoder we used a design with one input, six shift registers and two adders complying with industry standard rate of $1 / 2$ [1]. The simulation system that we developed produced from 64 to 8192 subcarriers in IFFT output. Table characteristics of up to 4096 subcarriers system were used from our previous study on noise effects in large number of subcarriers [11]. Simulation testbded of 8192 subcarriers in IFFT output was conformed accordingly to table structure of previous paper. Our system design appears in Figure 1.

\section{Soft Reduction Combined with $\mu$-Law}

The proposed scheme $\mu$-Law Soft Reduction $-\mu$ LSR introduces the attachment of a new compressor after $\mathrm{Cy}$ clic Prefix function. Companded output can be represented by following equations without the need of expanding it in receiver's part:

$$
\mu \mathrm{LSR}_{\text {out }}=V_{S R} \frac{\log \left(1+\mu \frac{\left|x_{S R}\right|}{V_{S R}}\right)}{\log (1+\mu)} \operatorname{sgn}\left(x_{S R}\right)
$$

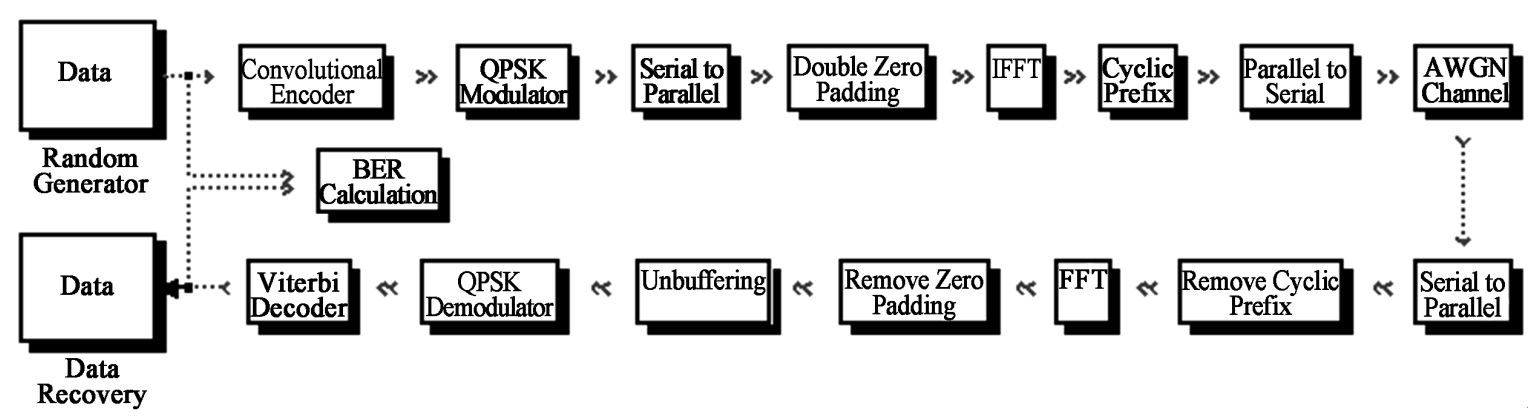

Figure 1. OFDM system overview. 


$$
x_{S R}=\frac{C P_{\text {out }}}{\left(1+C P_{\text {out }}^{2 P}\right)^{1 / 2 P}}
$$

where, $x_{S R}$ is Soft Reduction output, $P$ and $C P_{\text {out }}$ represent maximum peak and instantaneous signal amplitude in the output of Cyclic prefix block accordingly, $V_{S R}$ is the peak amplitude of Soft Reduction output and $\mu$ is the compression parameter.

By using Soft Reduction (SR) in the output of Cyclic Prefix, signal peaks which exhibited larger values than others in relation to threshold, were attenuated in a greater extend [12]. PAPR decreased along with the peak-topeak amplitude of the $C P_{\text {out }}$. This led us to connecting this algorithm with $\mu$-Law compression technique for PR $=2$ [13]. Result was the amplification of the reduced signal $\mathrm{x}_{\mathrm{SR}}$, while giving gain superiority to lower amplitudes and decreasing even more PAPR. The $\mu$-parameter was chosen to be equal to 3 for avoiding BER deterioration in no presence of ADCs and DACs. Figure 2 presents a graphical step by step function of $\mu$-Law Soft Reduction algorithm.

Also, in Figure 3, BER performance of $\mu \mathrm{LSR}$ is compared to $\mathrm{SR}$, to $\mu$-Law, and to OFDM system without convolutional encoder. There is clearly shown that BER performance of the proposed scheme is identical to that of SR and $\mu$-Law compressor $(\mu=3, \mathrm{PR}=2)$ with the

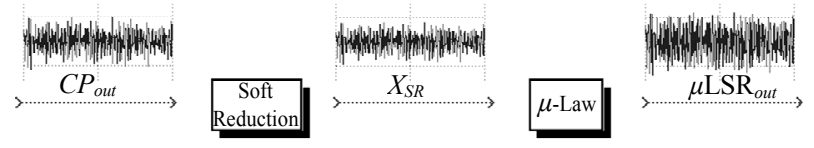

Figure 2. $\mu$-Law soft reduction compressor (time domain).

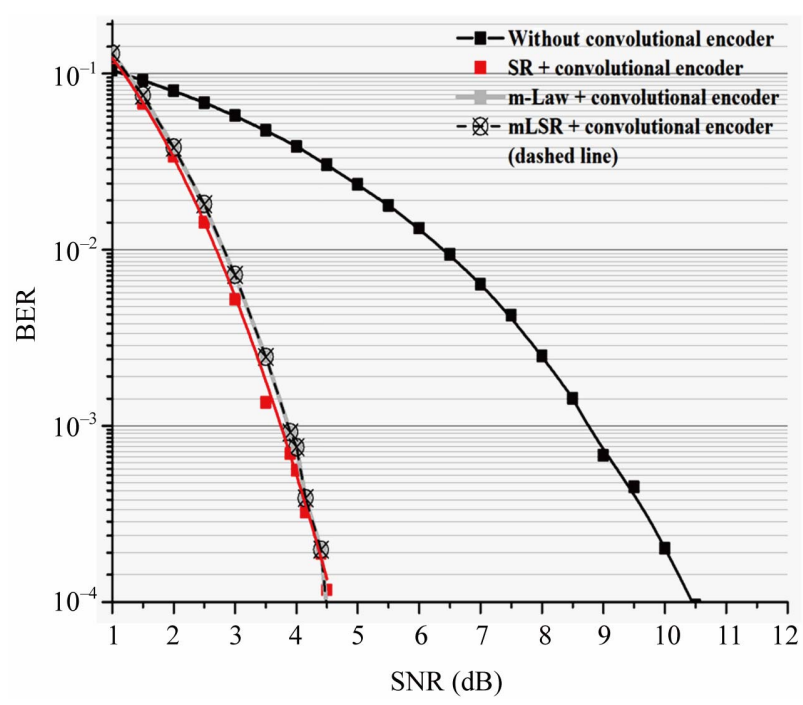

Figure 3. BER Performance of $\mu$ LSR for 8192 subarriers (IFFT output). System exhibited similar performance for 64, 128, 512, 1024, 2048 and 4096 subcarriers. presence of a convolutional encoder. BER deterioration was obviated. Almost 4.5 dB SNR is needed for sustaining data transferring with error percentage of $0.01 \%$. PAPR simulations of $\mu \mathrm{LSR}$ are mentioned in fifth section where a comparison is made between proposed and already known techniques.

\section{Subtracting Cyclic Prefix Output from $\mu$-Law Output}

The proposed scheme $\mu$-Law output subtracting Cyclic Prefix output $-\mu \mathrm{LaCP}$ is based again on the idea that $\mu$-Law compressor is placed after Cyclic Prefix section. But, further sinking of PAPR is imminent as primary signal $\left(C P_{\text {out }}\right)$ is deducted from the already amplified signal ( $\mathrm{PR}=2, \mu=3$, with reduced PAPR) [14]. In turn, this produces an output with lower power levels which doesn't pose any constraints in the final system design. The reason is that amplification in original power levels can be accomplished easily by using a gain block. In our simulations we didn't implement the previous block in order to keep low complexity in system design. Equations (3) and (4) describe mathematically the proposed method, while Figure 4 provides a step by step time domain graphical representation of $C P_{\text {out }}, \mu-\mathrm{Law}_{\text {out }}$ and $\mu \mathrm{LaCP}_{\text {out }}$ behavior.

$$
\begin{gathered}
\mu \mathrm{LaCP}_{\text {out }}=\mu \mathrm{L} \operatorname{sgn}\left(C P_{\text {out }}\right)-C P_{\text {out }} \\
\mu \mathrm{L}=\mathrm{PR} C P_{P_{\text {EAK }}} \frac{\log \left(1+\mu \frac{\left|C P_{\text {out }}\right|}{\operatorname{PR} C P_{\text {PEAK }}}\right)}{\log (1+\mu)}
\end{gathered}
$$

where, $C P_{\text {out }}$ is Cyclic Prefix output, $C P_{P E A K}$ represents maximum peak amplitude in the output of Cyclic prefix block, $\mu$ is the compression parameter and PR is the Peak Ratio [14].

In Figure 5, BER performance of $\mu \mathrm{LaCP}$ is compared to $\mathrm{SR}$, to $\mu$-Law, and to OFDM system without convolutional encoder. BER deteriorates slightly, because an additional mean value of $0.5 \mathrm{~dB}$ SNR is needed for accomplishing $0.01 \%$ error percentage. Also, BER deteriorates by the same factor compared to $\mu \mathrm{LSR}$, which exhibits duplicate BER performance compared to other schemes like SR and $\mu$-Law. The main advantage of $\mu \mathrm{LaCP}$ compared to other schemes is shown in section 5 where PAPR simulations are discussed in depth.

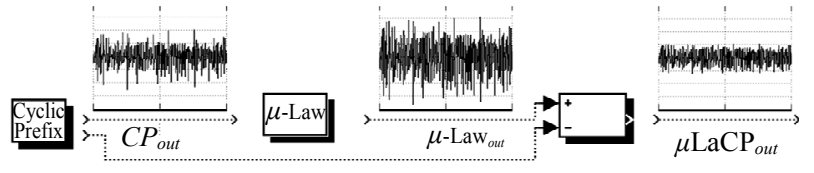

Figure 4. $\mu$-Law output subtracting cyclic prefix output (Time domain). 


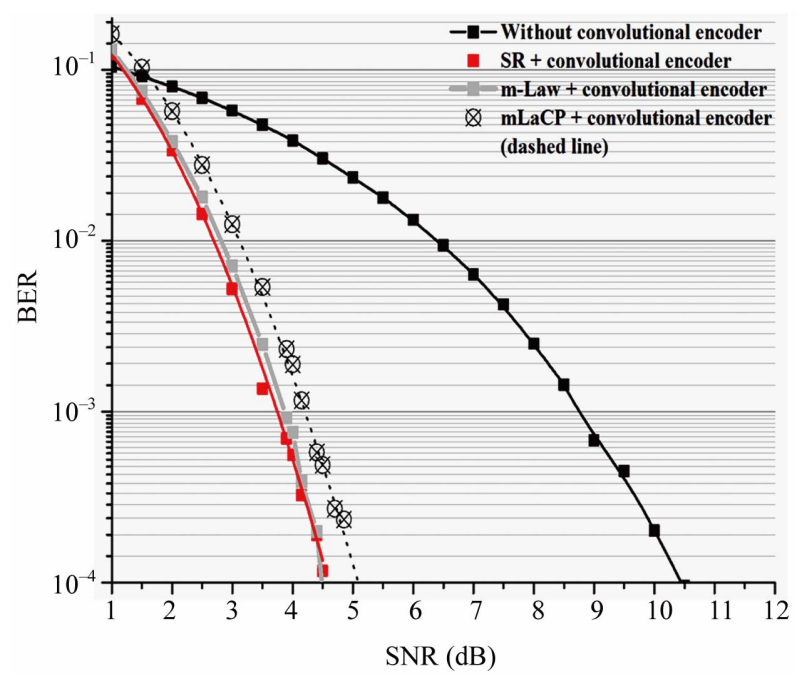

Figure 5. BER Performance of $\mu \mathrm{LaCP}$ for 8192 subarriers respectively (IFFT output). System exhibited similar performance for 64, 128, 512, 1024, 2048 and 4096 subcarriers.

\section{PAPR Simulations}

As previously mentioned, PAPR is a major problem in multicarrier transmission. We can define it as the ratio of the maximum instantaneous power to the average power.

$$
\mathrm{PAPR}=\max \frac{P(t)}{P_{\text {average }}}=\max \frac{|x(t)|^{2}}{E\left\{|x(t)|^{2}\right\}}
$$

In this section two sets of simulations are discussed. The first set is involved with finding the overall maximum PAPR that can be occurred. But, since this maximum PAPR rarely occurs, then the PAPR performance must be evaluated thoroughly using the Complementary $\mathrm{Cu}-$ mulative Distribution Function which relates directly with the second set of simulations. Assuming that all samples do not correlate with each other, the probability that PAPR ratio can be under a certain threshold is declared by Equation (6). Many researchers have involved with Distribution of OFDM PAPR. Van Nee proposed that Distribution of N carriers with oversampling, can be approximated with $\alpha N$ carriers without oversampling. Also, by taking into consideration that the effect of oversampling is approximated by inserting additional independent samples, he ended up in finding a new mathematical approach for CCDF [15].

$$
\begin{gathered}
P(\mathrm{PAPR} \leq z)=F(z)^{N}=\left(1-e^{-z}\right)^{N} \\
\mathrm{CCDF}=1-\left(1-e^{-z}\right)^{\alpha N}
\end{gathered}
$$

where $z$ corresponds to PAPR threshold level, $N$ is the number of carriers and $\alpha$ is given empirically as $\alpha=2.8$ for $N>64$.
Table 1 shows the absolute maximum PAPR for different number of total subcarriers. By using $\mu \mathrm{LSR}$ the maximum PAPR can be decreased even more compared to $\mu$-Law technique, varying between $15.2 \%$ and $0.6 \%$ for different number of subcarriers. Also, the comparison of $\mu \mathrm{LaCP}$ with $\mu$-Law reveals an innovative decrease varying from $52.3 \%$ to $48.3 \%$.

As maximum PAPR rarely occurs additional simulations had to be conducted with higher amount of information data. This was done in order to verify the innovative performance of the proposed methods through the calculation and plotting of CCDFs for 64 and 128 OFDM carriers. Actually, Figure 6 shows that $\mu$ LSR (for 64 carriers) has better performance compared to $\mu$-Law and to OFDM system with convolutional encoder, by almost $1 \mathrm{~dB}$ and $2.5 \mathrm{~dB}$ PAPR respectively (for theoretical maximum PAPR). Also $\mu$ LSR (for 128 carriers) exhibits PAPR decrease by almost $0.5 \mathrm{~dB}$ and $2.5 \mathrm{~dB}$ compared to previously mentioned systems. $\mu \mathrm{LaCP}$ has better

Table 1. Absolute maximum observed PAPR for different number of total subcarriers.

\begin{tabular}{ccccc}
\hline $\begin{array}{c}\text { Number of } \\
\text { subcarriers }\end{array}$ & $\begin{array}{c}\text { Only } \\
\text { convolutional } \\
\text { encoder }\end{array}$ & $\begin{array}{c}\text { Conv. } \\
\text { Encoder with } \\
\boldsymbol{\mu} \text {-Law }\end{array}$ & $\begin{array}{c}\boldsymbol{\mu L S R} \\
\text { scheme }\end{array}$ & $\boldsymbol{\mu L a C P}$ \\
\hline 81 & 10,95 & 7,41 & 6,28 & 3,68 \\
162 & 12,03 & 8,15 & 7,31 & 4,09 \\
324 & 13,87 & 7,89 & 7,09 & 4,42 \\
648 & 14,13 & 8,84 & 8,48 & 4,25 \\
1296 & 12,73 & 7,84 & 7,63 & 3,94 \\
2592 & 12,42 & 8,11 & 8,03 & 4,14 \\
5184 & 11,53 & 7,51 & 7,45 & 3,88 \\
10368 & 12,81 & 8,01 & 7,95 & 3,82 \\
\hline
\end{tabular}

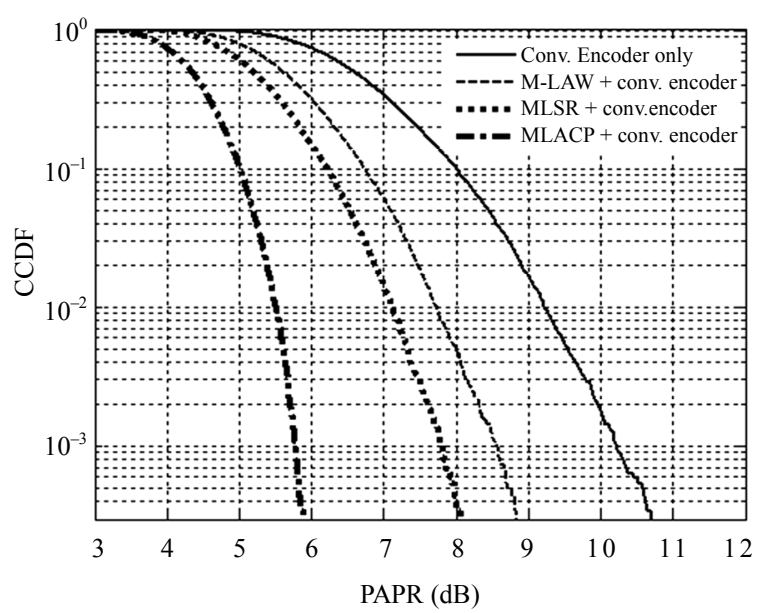

(a) 64 carriers 


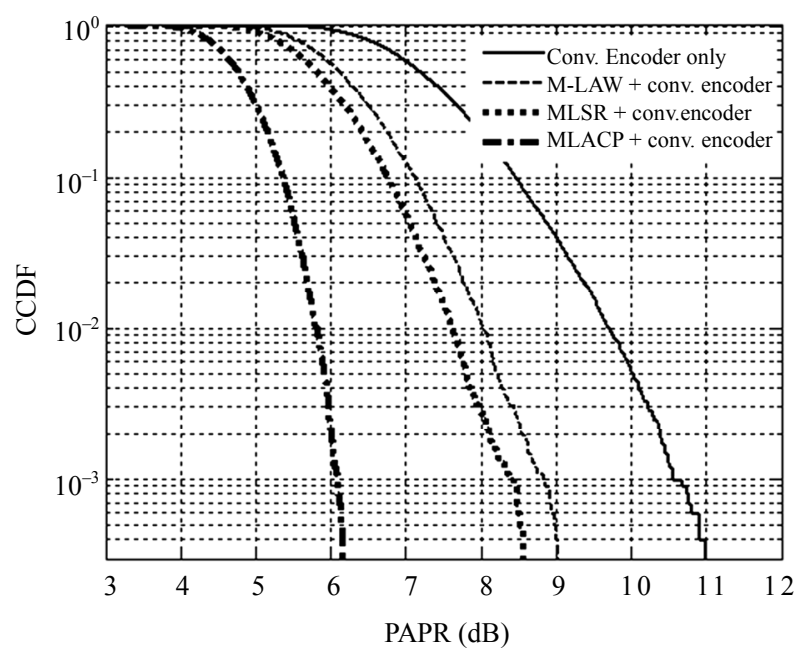

(b) 128 carriers

Figure 6. CCDF of the OFDM PAPR for 64 and 128 carriers (For all $\mu$-Law blocks: $\mu=3$ and PR $=2$ ).

performance compared to all other techniques. Maximum PAPR compared to $\mu \mathrm{LSR}, \mu$-Law and OFDM system with convolutional encoder, has been decreased by almost $2 \mathrm{~dB}, 3 \mathrm{~dB}$ and $4.5 \mathrm{~dB}$ respectively (for theoretical maximum PAPR). Hence, in order to understand $\mu \mathrm{LaCP}$ better performance compared to all other schemes, we can observe the probability of depressing PAPR under various values. For example, decreasing PAPR less than $5 \mathrm{~dB}$ for $\mu \mathrm{LaCP}, \mu \mathrm{LSR}, \mu$-Law and OFDM conv. system, concludes to a probability of $90 \%, 40 \%, 20 \%$ and below $1 \%$ respectively for 64 carriers - from Figure 6(a).

\section{Conclusion}

In this paper we proposed two new techniques for decreasing Peak to Average Power Ratio. The primary concern was to accomplish this with no BER deterioration and hence to keep complexity of the system as low as possible. BER curves for $\mu \mathrm{LSR}$ and $\mu \mathrm{LaCP}$ which were derived from simulations (in the absence of ADC and DAC) showed clearly not a severe deterioration. $\mu \mathrm{LSR}$ had a slightly better performance $(0.5 \mathrm{~dB})$ compared to $\mu \mathrm{LaCP}$, but the last exhibited a superior PAPR performance in terms of probability and maximum PAPR. Also, $\mu \mathrm{LaCP}$ is even simpler technique compared to $\mu \mathrm{LSR}$. Both techniques don't include an expander in receiver's part, for simplicity reasons. Additionally, these two new methods in their present forms give the capability in the final designer to implement them easily in a DSP. This is also a future goal of us, along with the design of a final OFDM system (vast number of subccarriers) introducing precise channel estimation.

\section{Acknowledgements}

The research Project is co-funded by the European Union - European Social Fund (ESF) \& National Sources, in the framework of the program "HRAKLEITOS II" of the "Operational Program Education and Life Long Learning" of the Hellenic Ministry of Education, Life Long Learning and religious affairs.

\section{References}

[1] R. Van Nee, G. Awater, M. Morikura, H. Takanashi, M. Webster and K. W. Halford, "New High-Rate Wireless LAN Standards," IEEE Communications Magazine, Vol. 37, No. 12, December 1999, pp. 82-88. doi:10.1109/35.809389

[2] S. I. Hernandez, "Simulation and Evaluation of a DVB System Using Simulink (Vol. I)," Bachelor Thesis, Linkopings Universitet, Linkoping, April 2005.

[3] F. Zbynek and S. Vladimir, "Reduction of PAPR in OFDM by Clipping," Proceedings of 15th International Czech-Slovak Scientific Conference Radioelektronika, Brno, May 2005, pp. 474-477.

[4] A. Mobasher and A. K. Khadani "PAPR Reduction in OFDM Systems Using Constellation Shaping," Proceedings of the 22nd Biennial Symposium on Communications, Kingston, May 31-June 3, pp. 258-260.

[5] R. Z. Luo, L.J. Jing, L. Luo, J.Li and W.L. Zhu, "Reducing the Peak-to-Average Power Ratio of OFDM System with Low Complexity," Journal of Electronics (China), Vol. 23, No. 1, January 2006, pp. 26-28. doi:10.1007/s11767-004-0030-5

[6] A. A. Eltholth, A. R. Mikhail, A. Elshirbini, M. I. Moawad and A. I. Abdelfattah, "Peak-to-Average Power Ratio Reduction in OFDM Systems Using Huffman Coding," World Academy of Science, Engineering and Technology, Vol. 43, July 2008, pp. 266-270.

[7] I. I. Al-kebsi, M. Ismail, K. Jumari and T. A. Rahman, "Eliminate the Effects of Clipping Technique on the SER Performance by Recovering the Clipped Part of the OFDM Signal," International Journal of Computer Science and Network Security, Vol. 9, No. 7, July 2009, pp. $37-45$.

[8] P.-H. Chang, S.-S. Jeng and J.-M. Chen, "Utilizing a Novel Root Companding Transform Technique to Reduce PAPR in OFDM Systems," International Journal of Communication Systems, Vol. 23, No. 4, February 2010, pp. 447-461.

[9] H. Sakran, M. Shokair and A. A. Elazm, "Combined Interleaving and Companding for PAPR Reduction in OFDM Systems," Progress in Electromagnetics Research $C$, Vol. 6, 2009, pp. 67-78.

[10] A. Vallavaraj, B. G. Stewart, D. K. Harrison and F. G. McIntosh, "The Effects of Convolutional Coding on BER Performance of Companded OFDM Signals," Proceedings of International Conference on Communication, Computer \& Power, Muscat, 14-16 February 2005, pp. 
201-204.

[11] S. K. Chronopoulos, C. Votis, V. Raptis, G. Tatsis and P. Kostarakis, "In Depth Analysis of Noise Effects in Orthogonal Frequency Division Multiplexing Systems, Utilising a Large Number of Subcarriers," 7th International Conference of the Balkan Physical Union, American Institute of Physics Conference Series, Vol. 1203, 2010, pp. 967-972.

[12] A. P. Pastor, "Simulation and Evaluation of a DVB System Using Simulink (Vol. II)," Bachelor Thesis, Linkopings Universitet, Linkoping, April 2005.

[13] A. Vallavaraj, B. G. Stewart, D. K. Harrison and F. G.
McIntosh, "Reduction of Peak to Average Power Ratio of OFDM Signals Using Companding," Proceedings of the 9th IEEE International Conference on Communications Systems, Singapore, 6-8 September 2004, pp. 160-164.

[14] A. Vallavaraj, B. G. Stewart and D. K. Harrison, "An Evaluation of Modified $\mu$-Law Companding to Reduce the PAPR of OFDM Systems," AEU-International Journal of Electronics and Communications, Vol. 64, No. 9, July 2009, pp. 844-857.

[15] R. Van Nee and R. Prasad, "OFDM for Wireless Multimedia Communications," Artech House, Boston, 2000, pp. 120-123. 